\title{
Indocyanine green fluorescent image-guided inguinal sentinel lymph node biopsy in vulvar cancer
}

Young Hwa Kwak, MD ${ }^{1,2}$, Yong Jae Lee, MD ${ }^{1,2}$, Jung-Yun Lee, MD, PhD ${ }^{1,2}$, Eun Ji Nam, MD, PhD ${ }^{1,2}$, Sunghoon Kim, MD, PhD ${ }^{1,2}$, Young Tae Kim, MD, PhD ${ }^{1,2}$, Sang Wun Kim, MD, PhD ${ }^{1,2}$

${ }^{1}$ Women's Cancer Center, Yonsei Cancer Center, ${ }^{2}$ Department of Obstetrics and Gynecology, Institute of Women's Life Medical Science, Yonsei University College of Medicine, Seoul, Korea

\section{Objective}

To demonstrate near-infrared fluorescence image-guided inguinal sentinel lymph node (SLN) biopsy in patients with vulvar cancer.

\section{Methods}

A 40-year-old woman with a 3-cm-sized palpable left vulvar mass was diagnosed with vulvar cancer on biopsy with protrusion into the vaginal cavity. Pelvic contrast-enhanced magnetic resonance imaging and F-18 fluorodeoxyglucose positron-emission tomography-computed tomography showed a small ulcerative enhancing lesion confined to the left vulva without distant metastasis. The patient was scheduled for radical vulvectomy with a left inguinal SLN biopsy. Indocyanine green was injected directly into the vulvar mass to map lymphatic drainage. A 4-cm-sized linear incision was made on the left inguinal crease, and the lymphatic channels of the left inguinal area were dissected under fluorescent image guidance using a 1588 Advanced Imaging Modalities Platform laparoscopic camera (Stryker, Kalamazoo, MI, USA).

\section{Results}

Fluorescence image-guided left inguinal SLN biopsy and radical vulvectomy were performed. The pathologic diagnosis confirmed vulvar adenoid cystic carcinoma with metastasis to the left inguinal lymph node (International Federation of Gynecology and Obstetrics stage IIIA). The patient was discharged without complications and received adjuvant radiotherapy.

\section{Conclusion}

This video demonstrates a successful ICG fluorescence image-guided left inguinal SLN biopsy in a vulvar cancer patient using a laparoscopic camera. Mapping of inguinal SLNs in patients with vulvar cancer may help in retaining surgical radicality while minimizing operative complications.

Keywords: Vulva cancer; Sentinel lymph node biopsy; Indocyanine green; Fluorescence

Received: 2021.11.08. Accepted: 2021.11.17.

Corresponding author: Sang Wun Kim, MD, PhD

Department of Obstetrics and Gynecology, Institute of Women's Life Medical Science, Yonsei University College of Medicine, 50-1 Yonseiro, Seodaemun-gu, Seoul 03722, Korea

E-mail: san1@yuhs.ac

https://orcid.org/0000-0002-8342-8701

\footnotetext{
Jung-Yun Lee and Young Tae Kim have been an Editorial Board of Obstetrics \& Gynecology Science; however, they are not involved in the peer reviewer selection, evaluation, or decision process of this article. Otherwise, no other potential conflicts of interest relevant to this article were reported. 


\section{Obstetrics \& Gynecology Science}

Vol. 65 , No. 2, 2022

Inguinal lymph node status is the most significant prognostic factor in patients with vulvar cancer [1]. The classical gold standard surgical treatment for patients with vulvar cancer is radical vulvectomy and complete inguinofemoral lymphadenectomy. Complete inguinofemoral lymph node dissection results in short- and long-term morbidities, including wound infections, dehiscence, and lymphedema [2]. Several studies have demonstrated that sentinel lymph node (SLN) biopsy significantly reduces complications while avoiding unnecessary lymphadenectomy, and has a low inguinal recurrence rate $[3,4]$.

SLN mapping using indocyanine green (ICG) with nearinfrared fluorescence imaging is a promising staging alternative in gynecologic malignancies and has been validated in patients with endometrial and cervical cancers $[5,6]$. Based on this evidence, this technique has been extended to vulvar cancers. Recently, several studies have shown the feasibility, accuracy, and safety of SLN mapping using ICG with nearinfrared fluorescence imaging in vulvar cancer $[7,8]$.

A 40-year-old woman was referred to our hospital for cancer in the left vulva that was diagnosed at another hospital in March 2020. She had no specific medical history other than two induced abortions. The vulvar biopsy specimen was reviewed by a gynecologic pathologist at our institute; adenoid cystic carcinoma was subsequently diagnosed. A baseline study was performed: Pap smear results showed adenoid cystic carcinoma, human papillomavirus genotyping revealed negative findings, and tumor markers were within the normal range. Examination of the vulvar mass lesion by colposcopy revealed an ulcerative and palpable mass, approximately $3 \mathrm{~cm}$ in size, in the left vulva. Pelvic contrast-enhanced magnetic resonance imaging showed small ulcerative enhancing lesions of approximately $3.4 \mathrm{~cm}$ in the left vagina; these were predicted to be International Federation of Gynecology and Obstetrics (FIGO) stage II. F-18 fluorodeoxyglucose (FDG) positron-emission tomography-computed tomography showed mild FDG uptake in the left vulva area, suggesting postoperative change; however, residual tumors could not be excluded. In both images, there was no evidence of metastasis to the lymph nodes or other organs. Esophagogastroduodenoscopy, colonoscopy, and breast sonography revealed no tumors in the upper gastrointestinal tract, colon, or breast, respectively. Under the diagnosis of vulvar cancer, the patient was scheduled to undergo radical vulvectomy and ICG fluorescent image-guided inguinal SLN biopsy. Approximately 3
$\mathrm{mL}$ of ICG $(1.25 \mathrm{mg} / \mathrm{mL})$ was directly injected into the vulvar mass for lymphatic drainage mapping. A 4-cm linear incision was made on the left inguinal crease, lymphatic channels were dissected, and an SLN was identified using the 1588 Advanced Imaging Modalities Platform laparoscopic camera (Stryker, Kalamazoo, MI, USA). After removing the left inguinal SLN, the node was sent out for frozen section diagnosis, which revealed few atypical cell clusters. The operation was completed after radical vulvectomy with an adequate resection margin. However, the final pathology result showed metastasis to the left inguinal SLN, indicating vulvar cancer, FIGO stage IIIA. The patient then received radiation therapy to the inguinal area and was monitored for 16 months without recurrence.

The advantages of SLN mapping using ICG with near-infrared fluorescence imaging include easier application, absence of radioactivity, and fewer side effects [8]. In addition, the procedure is less invasive, with little pain and scarring.

SLN biopsy is recommended as the standard treatment for women with early-stage vulvar cancer [9]. Comba et al. [10] showed that videoendoscopic SLN biopsy in vulvar cancer is a feasible procedure, and there may be increased usage of SLNs in early stage vulvar cancer with a videoendoscopic approach. However, this case demonstrated that the use of ICG with near-infrared fluorescence imaging for SLN biopsy is effective even at advanced stages. Gu et al. [11] reported that appropriate adjuvant radiotherapy SLN biopsy alone provided similar long-term survival to inguinofemoral lymphadenectomy for patients with or without sentinel node metastasis.

In conclusion, this video demonstrates that ICG fluorescent image-guided inguinal SLN biopsy using a laparoscopic fluorescence imaging system could be applied in vulvar cancer. Mapping of inguinal SLNs in vulvar cancer patients may help in retaining surgical radicality while minimizing operative complications.

\section{Conflict of interest}

No potential conflict of interest relevant to this article was reported. 


\section{Obstetrics \& Gynecology Science}

Young Hwa Kwak, et al. Sentinel lymph node biopsy in vulva cancer

\section{Ethical approval}

This study does not require approval of the Institutional Review Board because no patient data is contained in this article. The study was performed in accordance with the principles of the Declaration of Helsinki.

\section{Patient consent}

This article has not been previously presented.

\section{Funding information}

None.

\section{Acknowledgements}

This video has not been published elsewhere, and it is not accepted for publication or under review for publication in any other journal.

\section{Video clip}

Video can be found with this article online at https://doi. org/10.5468/ogs.21335.

\section{References}

1. Burger MP, Hollema $H$, Emanuels AG, Krans M, Pras E, Bouma J. The importance of the groin node status for the survival of T1 and T2 vulval carcinoma patients. Gynecol Oncol 1995;57:327-34.

2. Hinten F, van den Einden LC, Hendriks JC, van der Zee AG, Bulten J, Massuger LF, et al. Risk factors for shortand long-term complications after groin surgery in vulvar cancer. Br J Cancer 2011;105:1279-87.

3. Erickson BK, Divine LM, Leath CA 3rd, Straughn JM Jr. Cost-effectiveness analysis of sentinel lymph node bi- opsy in the treatment of early-stage vulvar cancer. Int J Gynecol Cancer 2014;24:1480-5.

4. Te Grootenhuis NC, van der Zee AG, van Doorn HC, van der Velden J, Vergote I, Zanagnolo V, et al. Sentinel nodes in vulvar cancer: long-term follow-up of the GROningen international study on sentinel nodes in vulvar cancer (GROINSS-V) I. Gynecol Oncol 2016;140:814.

5. Frumovitz M, Plante M, Lee PS, Sandadi S, Lilja JF, Escobar PF, et al. Near-infrared fluorescence for detection of sentinel lymph nodes in women with cervical and uterine cancers (FILM): a randomised, phase 3, multicentre, non-inferiority trial. Lancet Oncol 2018;19:1394-403.

6. Buda A, Crivellaro C, Elisei F, Di Martino G, Guerra L, De Ponti $\mathrm{E}$, et al. Impact of indocyanine green for sentinel lymph node mapping in early stage endometrial and cervical cancer: comparison with conventional radiotracer $(99 \mathrm{~m}) \mathrm{Tc}$ and/or blue dye. Ann Surg Oncol 2016;23:2183-91.

7. Koual M, Benoit L, Nguyen-Xuan HT, Bentivegna E, Azaïs $H$, Bats AS. Diagnostic value of indocyanine green fluorescence guided sentinel lymph node biopsy in vulvar cancer: a systematic review. Gynecol Oncol 2021;161:436-41.

8. Siegenthaler F, Imboden S, Knabben L, Mohr S, Papadia A, Mueller MD. Exploratory study of the clinical value of near-infrared sentinel lymph node mapping with indocyanine green in vulvar cancer patients. Front Oncol 2021;11:652458.

9. Covens A, Vella ET, Kennedy EB, Reade CJ, Jimenez W, Le T. Sentinel lymph node biopsy in vulvar cancer: systematic review, meta-analysis and guideline recommendations. Gynecol Oncol 2015;137:351-61.

10. Comba C, Erdogan SV, Erdogan A, Bagdatli A, Atas BS, Demir $O$, et al. Videoendoscopic sentinel lymph node detection with icg and bilateral inguinofemoral lymphadenectomy in vulvar cancer comba-ozdemir technique. Obstet Gynecol Sci 2021;64:552-4.

11. Gu H, Liu G, Li J, Chen J, Zhang X, Liu Z, et al. Longterm outcomes of individualized management after sentinel lymph-node biopsy for vulvar cancer. Int J Clin Oncol 2021;26:784-93. 